\title{
CORRESPONDENGE
}

\section{The Treatment of Well-Differentiated Thyroid Carcinoma}

by Prof. Dr. med. Ralf Paschke, Dr. med. Thomas Lincke, Dr. med. Stefan P. Müller, PD Dr. med. Michael C Kreissl, Prof. Dr. med. Dr. h. c. Henning Dralle, Prof. Dr. Martin Fassnacht in issue 26/2015

\section{Extent of Lateral Neck Dissection in Differentiated Thyroid Carcinoma}

In the surgical part of this review (1), the classification of cervical lymphadenectomies is restricted to central and lateral neck dissections. However, it is rather accurate and relevant to further break down and refine the latter term, as the surgical morbidity associated with classic radical or modified radical neck dissections can be significantly reduced by offering standardized, functional and selective neck dissections instead.

Similarly to other head and neck cancers, the American Thyroid Association (ATA) recommends the classification of the American Head and Neck Society, to precisely identify neck levels from I to VII, with additional side designation (2). Of these, levels I to $\mathrm{V}$ are included in a comprehensive lateral neck dissection, while levels VI and VII make up a central neck dissection. In cases with lateral lymph node involvement, where the metastatic burden is limited to levels III and IV, a selective dissection of levels IIa, III, IV, and Vb is recommended (2).

Further, there is evidence that the lymph node ratio (LNR), defined as the number of positive lymph nodes (numerator) divided by the total number of removed lymph nodes (denominator), is an independent prognostic factor (3). This is why it is paramount to achieve oncologic completeness by removing all lymph nodes from the selectively targeted neck levels while leaving all nonlymphatic structures intact, as this result has prognostic implications. The denominator of LNR, also known as nodal yield, depends - among other factors - on the applied surgical technique (4).

DOl: $10.3238 / a r z t e b l .2015 .0722 a$

\section{REFERENCES}

1. Paschke R, Lincke T, Müller SP, KreissI MC, Dralle H, Fassnacht M: The treatment of well-differentiated thyroid carcinoma. Dtsch Arztebl Int 2015; 112: 452-8.

2. Stack BC, Ferris RL, Goldenberg D, et al.: American Thyroid Association consensus review and statement regarding the anatomy, terminology, and rationale for lateral neck dissection in differentiated thyroid cancer. Thyroid 2012; 22: 501-8.

3. Vas Nunes JH, Clark JR, Gao K, et al.: Prognostic implications of lymph node yield and lymph node ratio in papillary thyroid carcinoma. Thyroid 2013; 23: 811-6.

4. Lorincz BB, Langwieder F, Mockelmann N, Sehner S, Knecht R: The impact of surgical technique on neck dissection nodal yield: making a difference. Eur Arch Otorhinolaryngol. 2015 Mar 18 [Epub ahead of print]

Dr. med. Balazs B. Lörincz, Ph.D.

Prof. Dr. med. Rainald Knecht

Universitäts-Klinik für HNO-Heilkunde, Kopf-Hals-Chirurgie und Onkologie

Universitätsklinikum Hamburg-Eppendorf

r.knecht@uke.de

\section{In Reply:}

Dralle (1) developed the technique of compartment-oriented microdissection of the central and lateral compartments in patients with thyroid carcinoma in the early 1990s; this technique then became a consensus-backed part of the guidelines (2). While today prophylactic compartment resection has generally been abandoned in patients with intrathyroid papillary and follicular cN0 carcinoma in whom preoperatively or intraoperatively no lymph-node metastasis is suspected, there is a consensus that compartment-oriented lymph-node dissection should be performed where therapeutically indicated. With regard to the extent of lateral compartment resection, the specialist societies $(1,2)$ draw particular attention to the risk of morbidity associated with the nerves and the thoracic duct located in the lateral compartment and recommend that the extent of the resection should depend on tumor involvement (Table 2, in [3]). Thus, in patients with no signs of lymph-node metastasis, it is acceptable to not perform lymph-node dissection to minimize the risk of intraoperative injury to the marginal mandibular branch of the facial nerve or to the accessory nerve to prevent associated functional impairments (2).

For papillary carcinoma, it has long been proven that the extent of lymph-node involvement is a prognostic risk factor, especially for the risk of distant metastasis (4). The strategy of compartment resection is based on this. Whether lymph-node micrometastasis as opposed to lymph-node macrometastasis has a significant impact on survival beyond its role as a risk indicator remains unclear. Based on the current consensus of the specialist societies, the individualized risk/benefit assessment with regard to the extent of compartment resection favors a tumor involvement-oriented resection strategy, taking into account local macroscopic and, in case of distant metastasis, also distant tumor involvement.

DOI: 10.3238/arztebl.2015.0722b

\section{REFERENCES}

1. Dralle H, Musholt TJ, Schabram J, et al.: German Association of Endocrine Surgeons practice guideline for the surgical management of malignant thyroid tumors. Langenbecks Arch Surg 2013; 398: 347-75.

2. Stack BC, Ferris RL, Goldenberg D, et al.: American thyroid association consensus review and statement regarding the anatomy, terminology, and rationale for lateral neck dissection in differentiated thyroid cancer. Thyroid 2012; 22: 501-8.

3. Paschke R, Lincke T, Müller SP, KreissI MC, Dralle H, Fassnacht M: The treatment of well-differentiated thyroid carcinoma. Dtsch Arztebl Int 2015; 112: 452-8.

4. Machens A, Dralle H: Correlation between the number of lymph node metastases and lung metastasis in papillary thyroid cancer. J Clin Endocrinol Metab 2012; 97: 4375-82

\section{Prof. Dr. med. Ralf Paschke}

Klinik für Endokrinologie und Nephrologie, Universitätsklinikum Leipzig ralf.paschke@medizin.uni-leipzig.de

Prof. Dr. med. Dr. h. c. Henning Dralle

Universitätsklinik und -Poliklinik für Allgemein-, Viszeral- und Gefäßchirurgie Universitätsklinikum Halle (Saale)

\section{Conflict of interest statement}

Prof. Paschke has received consultancy fees, reimbursement of expenses for conference participation and travel and accommodation expenses from Bayer and Eisai. He received fees for commissioned clinical trials into an external funding account from Bayer, Eisai and AstraZeneca. Prof. Dralle declares that no conflict of interests exists. 